\title{
Intestinal endometriosis: a diagnostic dilemma
}

\author{
Sunil Kumar Juneja ${ }^{1}$, Satpal Singh Virk ${ }^{2}$, Saurabh Singla ${ }^{2}$, Kamal Sachdeva ${ }^{2 *}$, \\ Harpreet Kaur ${ }^{3}$, Anjali Sharma ${ }^{1}$
}

\begin{abstract}
${ }^{1}$ Department of Obstetrics and Gynecology, ${ }^{2}$ Department of Gastrosurgery, ${ }^{3}$ Department of Pathology, Dayanand Medical College and Hospital, Ludhiana, Punjab, India
\end{abstract}

Received: 01 August 2018

Accepted: 16 January 2019

\section{*Correspondence:}

Dr. Kamal Sachdeva,

E-mail: kamal2471987@gmail.com

Copyright: (C) the author(s), publisher and licensee Medip Academy. This is an open-access article distributed under the terms of the Creative Commons Attribution Non-Commercial License, which permits unrestricted non-commercial use, distribution, and reproduction in any medium, provided the original work is properly cited.

\begin{abstract}
Background: Endometriosis is a benign gynaecological condition that causes significant morbidity to women of reproductive age group. It uncommonly affects the gastrointestinal tract and acute bowel obstruction is a rare manifestation.

Methods: A retrospective observational study was conducted on eight patients.

Results: In three patients (37.5\%), small intestinal involvement was seen, and colon was involved in five patients $(62.5 \%)$. One $(12.5 \%)$ patient presented with complete bowel obstruction while in others partial obstruction of the intestine was seen.

Conclusions: Intestinal endometriosis is a diagnostic challenge and should be considered in young menstruating women with gastrointestinal symptoms. Intestinal endometriosis is the most common extra-pelvic site and it is found in $12 \%$ of women with endometriosis. The true incidence of endometriosis causing bowel obstruction is unknown. Pre or intraoperative sigmoidoscopy may prove helpful in ruling out malignancy. The gold standard for diagnosis is laparoscopy and biopsy, which allows a full assessment of the pelvis as well as surgical resection if required. The management of endometriosis is an integrated approach of both medical and surgical therapy. Bowel resection is usually undertaken if there are features of obstruction or bleeding, and if there is suspicion of malignancy.
\end{abstract}

Keywords: Bowel obstruction, Endometriosis, Intestinal endometriosis

\section{INTRODUCTION}

Endometriosis is the ectopic growth of viable endometrium outside the uterus, affecting approximately $7-10 \%$ of females. ${ }^{1}$ Endometriosis can involve intra- and extra-peritoneal sites. In decreasing order of frequency, the intra-peritoneal locations are ovaries (30\%), uterosacral ligaments (18\%-24\%), fallopian tubes $(20 \%)$, pelvic peritoneum, pouch of Douglas, and gastrointestinal (GI) tract. Extra-peritoneal locations include cervical portion $(0.5 \%)$, vagina and rectovaginal septum, round ligament and inguinal hernia sac $(0.3 \%-0.6 \%)$, umbilicus $(1 \%)$, abdominal scars after gynaecological surgery
$(1.5 \%)$ and caesarean section (0.5\%).Endometriosis rarely affects extra-abdominal organs such as the lungs, urinary system, skin and the central nervous system. ${ }^{2,3}$ TVS is the mainstay in evaluating symptoms associated with endometriosis and is accurate in detecting endometriomas. Gastrointestinal involvement of endometriosis has been estimated in 3\%-15\% of menstruating women. ${ }^{4}$ It is generally an asymptomatic condition and often diagnosed at operative time. The most common areas of intestinal involvement are the rectum and recto-sigmoid colon followed by sigmoid colon, cecum, terminal ileum, proximal colon and appendix. 
Endometriosis causing large intestinal obstruction is extremely rare with incidence less than one percent and is hard to differentiate from malignancy before surgery. ${ }^{4}$

\section{METHODS}

In accordance with the ethical principles and the approval by the institutional research and ethics committee a retrospective observational study was conducted in the Department of obstetrics and gynecology in collaboration with Department of Gastro-surgery of Dayanand medical college and hospital over the period of four years.

\section{Inclusion criteria}

- Women in reproductive age group with gastrointestinal symptoms like pain abdomen, bleeding per rectum, heaviness in lower abdomen menorrhagia and dysmenorrhea

- Women in reproductive any associated gynecological symptoms or signs were included in the study.

\section{Exclusion criteria}

- Pregnant women

- Post-menopausal women

- Blunt abdominal trauma in women of reproductive age group

- Any history of adnexal or uterine malignancy.

The relevant demographic data and findings were noted, and conclusion was derived.

\section{RESULTS}

In present study eight patients in the age group of 25-40 years presented with gastrointestinal symptoms without any specific positive family history.

They were operated on high suspicion and histopathological findings confirmed intestinal endometriosis.

Table 1: Data of patients of intestinal endometriosis admitted in Dayanand Medical College and Hospital.

\begin{tabular}{|l|l|l|l|l|}
\hline Age & Parity & Symptoms & Site involved & Procedure \\
\hline 25 & Nulliparous & Pain abdomen & Sigmoid colon & TAH with BSO with segmental sigmoid colectomy \\
\hline 29 & Multiparous & Pain abdomen & Sigmoid colon & No pelvic pathology with segmental sigmoid colectomy \\
\hline 33 & Multiparous & Pain abdomen & Sigmoid colon & Ovarian cystectomy with segmental sigmoid colectomy \\
\hline 34 & Multiparous & Pain abdomen & Ileal loops & Involved Ileal segment resection with ovarian cystectomy \\
\hline 30 & Multiparous & Pain abdomen & Ileal loops & No pelvic pathology \\
\hline 38 & Multiparous & Anemia & Sigmoid colon & Ovarian cystectomy with segmental sigmoid colectomy \\
\hline 40 & Multiparous & Bleeding per rectum & Sigmoid colon & Ovarian cystectomy with segmental sigmoid colectomy \\
\hline 40 & Multiparous & Pain abdomen & Ileal loops & Involved Ileal segment resection with ovarian cystectomy \\
\hline
\end{tabular}

In present study, only one $(12.5 \%)$ patient was nulliparous while others were multipara as shown in Table 1.

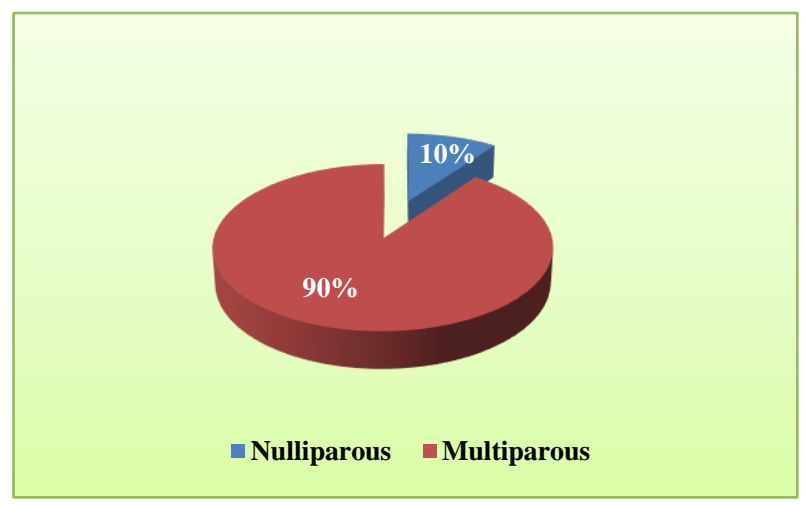

Figure 1: Pie chart correlates the relation of parity with endometriosis in present study.

Six $(75 \%)$ women presented with lower abdominal pain; one $(12.5 \%)$ with anemia and one $(12.5 \%)$ with bleeding per rectum. The small intestinal involvement was seen in three patients $(37.5 \%)$

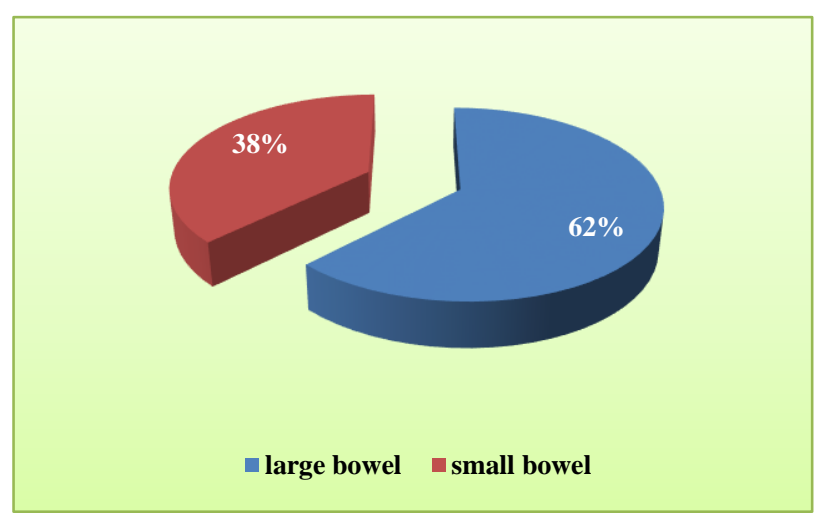

Figure 2: Pie-chart correlates the ratio of involvement of small bowel and large bowel in intestinal endometriosis in present study.

No identifiable pelvic pathology could be demonstrated in two out of the three women. In five $(62.5 \%)$ patients' 
colonic involvement was seen and in all these women chocolate cyst was found. One $(12.5 \%)$ patient presented with complete bowel obstruction while in others partial obstruction of the intestine was seen. Ovarian cystectomy was done for the patients who presented with chocolate cyst.

On close follow up, only one out of the operated cases of intestinal endometriosis represented with similar obstructive symptoms. Total abdominal hysterectomy and bilateral salpingo-oophorectomy was done for the patient.

\section{DISCUSSION}

Endometriosis is a clinical and pathological entity characterized by presence of functioning endometrial glands and stroma outside the uterine cavity. Endometriosis is a hormonally dependent disease and as a result is chiefly found in reproductive-aged women.

The incidence of endometriosis is difficult to quantify, as women with the disease are often asymptomatic, and imaging modalities shave low sensitivities for diagnosis. Many theories have been proposed to explain this condition; most common being Sampson's retrograde menstruation theory. ${ }^{5}$ Other theories include vascular dissemination, colonic metaplasia and autoimmune disease. $^{6}$

Women with endometriosis may be asymptomatic, sub fertile, or suffer varying degrees of pelvic pain. The classic triad of dysmenorrhea, dyspareunia and infertility is characteristic of the disease. Patients may present with chronic pelvic pain, constipation, diarrhea, hematochezia in case of gastrointestinal involvement.

Risk factors include:

- Women who have a first-degree relative (mother, sister, daughter) with the disease

- Women who are giving birth for the first time after 30 years

- White women

- Women with an abnormal uterus.

Intestinal endometriosis is the most common extra-pelvic site and it is found in $12 \%$ of women with endometriosis. ${ }^{7}$ It mostly affects the segments located within the pelvis, such as the terminal ileum, the appendix, the sigmoid colon and the rectum, both above and below the peritoneal reflection. The most common sites are the recto-sigmoid (up to $74 \%$ of cases) and recto-vaginal septum $(13 \%){ }^{8}$ Intestinal endometriosis are typically found in the serosa and muscularis layer, sparing the mucosa. ${ }^{6}$

Small intestinal involvement accounts for up to $16 \%$ of gastrointestinal endometriosis with cramping abdominal pain as the most common presenting symptom. ${ }^{4}$ In a radiologic study, Scarmato et al, detected endometriosis of the terminal and mid-ileum in four patients and one patient, respectively. ${ }^{9}$

Rarely, endometriosis proliferates, evolves and causes fibrosis and stricture of the intestine and thus causing bowel obstruction. ${ }^{6,7,10}$

The true incidence of endometriosis causing bowel obstruction is unknown. ${ }^{11}$ It occurs in less than one per cent of large intestinal endometriosis and in about $10 \%$ of small bowel involvement requiring surgery. ${ }^{4}$

It is a challenging condition to diagnose as intestinal endometriosis can manifest with acute and chronic symptoms that can mimic many different pathologies such as malignancy, inflammatory bowel disease, ischemic colitis, infectious diseases and IBS.

Pre or intraoperative sigmoidoscopy may prove helpful in ruling out malignancy. An intact mucosa effectively rules out primary colorectal malignancy. The gold standard for diagnosis is laparoscopy and biopsy, which allows a full assessment of the pelvis as well as surgical resection if required. ${ }^{12}$

The treatment of uncomplicated intestinal endometriosis depends on the patient's age and intention to conceive. Surgery is the treatment of choice for intestinal endometriosis in most cases. For the accidental finding without symptoms of obstruction, hormone therapy with danazol or gonadotrophin-releasing hormone (GnRH) analogs may be considered.

Bowel resection is usually undertaken if there are features of obstruction or bleeding, and if there is suspicion of malignancy. In acute colonic obstruction by endometriosis, it is important to confirm the underlying etiology as colonic malignancy will produce the same set of symptoms and requiring different surgical management.

In patients of child-bearing age, resection of the involved bowel followed by hormonal treatment may be sufficient; otherwise, hysterectomy and bilateral oophorectomy is the treatment of choice. In present study, biopsy of the intestinal site showed presence of endometrial glands and stroma present within muscularis propria and serosa and not involving mucosa; thus, confirming the diagnosis of intestinal endometriosis.

\section{CONCLUSION}

In conclusion, intestinal endometriosis is often a diagnostic challenge mimicking a broad spectrum of diseases and should be considered in young menstruating women with gastrointestinal symptoms.

\author{
Funding: No funding sources \\ Conflict of interest: None declared
}


Ethical approval: The study was approved by the Institutional Ethics Committee

\section{REFERENCES}

1. Parazzini F, Esposito G, Tozzi L, Noli S, Bianchi S. Epidemiology of endometriosis and its comorbidities. Eur J Obstet Gynecol Reprod Biol. 2017;209:3-7.

2. Frackiewicz EJ, Zarotsky V. Diagnosis and treatment of endometriosis. Expert Opin Pharmacother. 2003;4:67-82.

3. Lin YH, Kuo LJ, Chuang AY, Cheng TI, Hung CF. Extrapelvic endometriosis complicated with colonic obstruction. J Chin Med Assoc. 2006;69:47-50

4. Rock JA, Howard W, Telinde. Endometrosis. Operative Gynaecol. 2015;22:424-5.

5. Witz CA. Current concepts in the pathogenesis of endometriosis. Clin Obstet Gynecol.1999;42:566-85.

6. Katsikogiannis N, Tsaroucha A, Dimakis K, Sivridis E, Simopoulos C. Rectal endometriosis causing colonic obstruction and concurrent endometriosis of the appendix: a case report. J. Med. Case Rep. 2011;5:320.

7. Macafee $\mathrm{CH}$, Greer HL. Intestinal endometriosis. A report of 29 cases and a survey of the literature. J. Obstet. Gynaecol. Br. Emp. 2016;196067:539-5.
8. Tarjanne S, Sjöberg J, Heikinheimo O. Rectovaginal endometriosis-characteristics of operative treatment and factors predicting bowel resection. J Minim Invasive Gynecol. 2009;16(3):302-306.

9. Scarmato VJ, Levine MS, Herlinger H, Wickstrom M, Furth EE, Tureck RW. Ileal endometriosis: radiographic findings in five cases. Radiol. 2000;214:509-512.

10. Ribeiro PA, Rodrigues FC, Kehdi IP, Rossini L, Abdalla HS, Donadio N. Laparoscopic resection of intestinal endometriosis: a 5-year experience. J. Minim. Invasive Gynecol. 2006;13(5):442-6.

11. Paksoy M, Karabicak I, Ayan F, Aydogan F. Intestinal obstruction due to rectal endometriosis. Mt Sinai J Med. 2005;72:405-8.

12. Pramateftakis MG, Psomas S, Kanellos D, Vrakas G, Roidos G, Makrantonakis A. Large bowel obstruction due to endometriosis. Tech. Coloproctol. 2010;14(1):S87-9.

Cite this article as: Juneja SK, Virk SS, Singla S, Sachdeva K, Kaur H, Sharma A. Intestinal endometriosis: a diagnostic dilemma. Int J Reprod Contracept Obstet Gynecol 2019;8:1139-42. 